\title{
Prevalência e fatores de risco para transtornos de ansiedade em alunos de graduação da área da saúde de uma universidade pública do estado do Pará
}

\author{
Prevalence and risk factors for anxiety disorders in health care undergraduate students at a public \\ university in the state of Pará \\ Prevalencia y factores de riesgo de trastornos de ansiedad en estudiantes de pregrado en salud de \\ una universidad pública del estado de Pará
}

Laís Sena Leal

ORCID: https://orcid.org/0000-0002-2759-7594 Universidade do Estado do Pará, Brasil E-mail: lais.sena.leal@gmail.com

Regis Bruni Andriolo

ORCID: https://orcid.org/0000-0003-0306-5750 Universidade do Estado do Pará, Brasil E-mail:regis.andriolo@gmail.com

Lucivaldo da Silva Araújo

ORCID: https://orcid.org/0000-0002-8974-0886 Universidade do Estado do Pará, Brasil E-mail: lucivaldoaraujo@hotmail.com

\begin{abstract}
Resumo
Introdução: A vivência acadêmica traz desafios que podem, em determinados contextos, propiciar o surgimento dos transtornos de ansiedade. Objetivos: Verificar a prevalência de transtornos de ansiedade em graduandos dos cursos de saúde em uma Universidade Pública do Estado do Pará; verificar a relação entre transtorno de ansiedade, suporte social recebido, fatores socioeconômicos e demográficos. Metodologia: A pesquisa foi quantitativa analítica observacional transversal, realizada junto aos graduandos dos seguintes cursos das ciências da saúde: Terapia ocupacional, educação física, biomedicina, medicina e fisioterapia, através de pesquisa de campo, com aplicação de questionário online, no período de fevereiro a julho de 2021, utilizando como variável primária a aplicação da Escala de Hamilton para Avaliação da Ansiedade, e como variáveis secundárias, a Escala de Satisfação com o Suporte Social e a variável socioeconômica cultural, através de questionário. Resultados: No que se relaciona à maior prevalência dos transtornos de ansiedade detectou-se, que foi maior nos cursos de Terapia Ocupacional e Educação Física, maior junto aos acadêmicos que cursavam $3^{\circ}$ e $2^{\circ}$ anos, maior no sexo feminino, além disso, mostrou que quanto menor renda individual média dos acadêmicos, e os que contavam com menor suporte social recebido, maiores foram os escores para transtornos de ansiedade. Conclusão: A pesquisa revelou associações entre a prevalência dos transtornos de ansiedade nos cursos da área da saúde e os fatores que colaboram para este quadro. É imprescindível que os discentes e o corpo docente busque o melhor direcionamento do acadêmico a fim de prevenir os agravos associados a esta patologia.
\end{abstract}

Palavras-chave: Transtornos de ansiedade; Estudantes de ciências da saúde; Apoio social.

\begin{abstract}
Introduction: Academic experience brings challenges that can, in certain contexts, lead to the emergence of anxiety disorders. Objectives: To verify the prevalence of anxiety disorders in undergraduates of health courses at a Public University in the State of Pará; verify the relationship between anxiety disorder, social support received, socioeconomic and demographic factors. Methodology: The research was quantitative analytical observational transversal, carried out with undergraduates from the following health sciences courses: Occupational therapy, physical education, biomedicine, medicine and physiotherapy, through field research, with application of an online questionnaire, in February to July 2021, using the Hamilton Anxiety Assessment Scale as the primary variable, and the Social Support Satisfaction Scale and the socioeconomic cultural variable as secondary variables, through a questionnaire. Results: With regard to the higher prevalence of anxiety disorders, it was detected that it was higher in Occupational Therapy and Physical Education courses, higher among students attending the 3rd and 2nd years, higher in females, moreover, it showed that the lower average individual income of students, and those who had less social support received, the higher were the scores for anxiety disorders. Conclusion: The research revealed associations between the prevalence of anxiety disorders in healthcare courses and the factors that contribute to this situation. It is
\end{abstract}


essential that students and faculty seek the best guidance from the academic in order to prevent the problems associated with this pathology.

Keywords: Anxiety disorders; Students, health occupations; Social support.

\begin{abstract}
Resumen
Introducción: La experiencia académica conlleva desafíos que pueden, en determinados contextos, conducir a la aparición de trastornos de ansiedad. Objetivos: Verificar la prevalencia de trastornos de ansiedad en estudiantes de pregrado de cursos de salud en una Universidad Pública del Estado de Pará; verificar la relación entre trastorno de ansiedad, apoyo social recibido, factores socioeconómicos y demográficos. Metodología: La investigación fue observacional analítica cuantitativa transversal, realizada con estudiantes de pregrado de los siguientes cursos de ciencias de la salud: Terapia ocupacional, educación física, biomedicina, medicina y fisioterapia, a través de investigación de campo, con aplicación de un cuestionario en línea, de febrero a julio de 2021, utilizando la Escala de Evaluación de Ansiedad de Hamilton como variable primaria, y la Escala de Satisfacción de Apoyo Social y la variable socio-económico-cultural como variables secundarias, a través de un cuestionario. Resultados: Con respecto a la mayor prevalencia de trastornos de ansiedad, se detectó que fue mayor en los cursos de Terapia Ocupacional y Educación Física, mayor entre los estudiantes de $3^{\circ}$ y $2^{\circ}$ año, mayor en el sexo femenino, además, mostró que el menor promedio individual ingresos de los estudiantes, y aquellos que tenían menos apoyo social recibido, mayores eran las puntuaciones para los trastornos de ansiedad. Conclusión: La investigación reveló asociaciones entre la prevalencia de trastornos de ansiedad en los cursos de salud y los factores que contribuyen a esta situación. Es fundamental que estudiantes y profesores busquen la mejor orientación del académico para prevenir los problemas asociados a esta patología.
\end{abstract}

Palabras clave: Trastornos de ansiedad; Estudiantes de ciencias de la salud; Apoyo social.

\title{
1. Introdução
}

A vivência acadêmica é composta por inúmeras situações, demandas e desafios que podem, em determinados contextos sócio-econômico-culturais e pessoais, desencadear ou intensificar quadros de sofrimento psíquico que geralmente culminam na evasão discente (Gomes et al., 2018).

Como condição patológica, os transtornos de ansiedade, segundo critérios do DSM-V (Manual diagnóstico e estatístico das doenças mentais, do inglês, Diagnostic and Statistical Manual of Mental Disorders), incluem transtornos que compartilham características como medo e ansiedade excessivos e perturbações comportamentais relacionados. Medo é a resposta emocional à ameaça iminente real ou percebida, enquanto ansiedade é a antecipação de ameaça futura (APA, 2014).

Os transtornos de ansiedade se diferenciam do medo ou da ansiedade primária por serem excessivos ou persistirem além de períodos apropriados ao nível de desenvolvimento. Eles diferem do medo ou da ansiedade provisória, com frequência, induzidos por estresse, por serem persistentes (p. ex., em geral durando seis meses ou mais), embora o critério para a duração seja concebido como um guia geral, com a possibilidade de algum grau de flexibilidade, sendo às vezes, de duração mais curta (APA, 2014).

Durante a formação na universidade, o estudante está submetido a diferentes situações como por exemplo a rotina de estudos cada vez mais crescente, uma maior vulnerabilidade psicológica, podendo influenciar na mudança de comportamentos, desencadeando diversos tipos de transtornos, dentre eles, os transtornos de ansiedade, que podem se agravar com a presença de diversos estressores, como por exemplo excesso de tarefas acadêmicas, a falta de motivação para os estudos e a carreira escolhida, a existência de conflitos com colegas e professores, a apresentação de trabalhos, dificuldades na aquisição de materiais e livros, entre outros (Medeiros \& Bittencourt, 2016).

Em pesquisa realizada um Centro Universitário no Ceará obteve-se prevalências de ansiedade em torno de 36,1\% e de depressão próximo de 28,6\% entre os estudantes do primeiro ano dos cursos de Biomedicina, Enfermagem, Fisioterapia, Medicina e Odontologia (Leão et al., 2018).

Segundo a Associação Nacional dos Dirigentes da Instituições Federais de Ensino Superior (ANDIFES) (2014) em parceria com Fórum Nacional de Pró-reitores de Assuntos Comunitários e Estudantis (FONAPRACE), 58\% dos estudantes de graduação que responderam os questionários de uma pesquisa que objetivou refletir sobre a saúde mental na universidade 
declararam sofrer de ansiedade; 44\% declararam desânimo e falta de vontade; 32\% declararam insônia e 10\% declararam pensamento suicida.

Conforme estudo prévio realizado na UEPA de Leal et al. (2010), em que foi visualizado a presença de transtornos de ansiedade na realidade de apenas um dos cursos da saúde do Centro de Ciências Biológicas e da Saúde (CCBS) - Medicina, já se alertava para a necessidade de explorar e ampliar a investigação sobre essa realidade, a qual consiste no objeto da presente pesquisa.

Considerando estes fatores, surgiu o seguinte questionamento: "Qual a prevalência e os fatores de risco do transtorno de ansiedade entre estudantes universitários da área da saúde em uma universidade pública do Estado do Pará?”

O presente estudo visa verificar a prevalência de transtornos de ansiedade em acadêmicos de graduação dos cursos de saúde em uma universidade pública do Estado do Pará, bem como verificar a relação entre transtorno de ansiedade, suporte social recebido, fatores socioeconômicos e demográficos.

\section{Metodologia}

A pesquisa foi executada na Coordenação de Registro e Controle Acadêmico (CRCA), dos Campus II no Centro de Ciências Biológicas e da Saúde, em Belém-PA, que gerencia os dados dos cursos: Medicina, Fisioterapia, Biomedicina e Terapia Ocupacional e do campus III, na mesma cidade, responsável pelos dados do curso de Educação Física. Assim como via internet através de formulário online (google forms) vinculadas à matrícula do discente.

O projeto foi aprovado pelo Comitê de Ética em Pesquisa (CEP) da UEPA sob o parecer com número de aprovação CAEE 28819620.7.0000.5174. Os participantes aceitaram voluntariamente participar do estudo o Termo de Consentimento Livre e Esclarecido (TCLE) após aceite do TCLE online (formato online).

A pesquisa realizada foi do tipo quantitativa analítica observacional transversal através de pesquisa de campo e análise estatística das variáveis, a pesquisa quantitativa é centrada na objetividade, "considera que a realidade só pode ser compreendida com base na análise de dados brutos, recolhidos com o auxílio de instrumentos padronizados e neutros" (Gerhardt \& Silveira, 2009, p. 33).

"Estudos analíticos observacionais partem da observação da realidade, sugerindo hipóteses, sendo os transversais os estudos que analisam a situação de uma população em um dado momento" (Aragão, 2013).

Este tipo de estudo é utilizado quando há a possibilidade de examinar a associação entre determinantes de alguma condição que se relaciona com um fenômeno coletivo (Lima \& Barreto, 2003).

As variáveis primárias foram constituídas pelos escores dos sintomas comuns de ansiedade, segundo Escala de Hamilton para Avaliação da Ansiedade a qual "compreende 14 grupos de sintomas, subdivididos em dois grupos, sete relacionados a sintomas de humor ansioso e sete relacionados a sintomas físicos de ansiedade" (Sougey, 1987 p. 49-53).

Os itens são avaliados por uma escala que varia de 0 a 4 de intensidade ( $0=$ ausente; $2=$ leve; 3 = média; 4 = máxima). A soma dos escores varia em uma pontuação de 0 a 56. A escala considera que quanto mais grave for a manifestação, maior será o número de sintomas característicos que se apresentam (Ito \& Ramos, 1998), tendo sido os limiares classificados da seguinte maneira: a) $\geq 18$, ansiedade leve; $b) \geq 25$, ansiedade moderada; $c) \geq 30$, ansiedade grave.

Já as variáveis secundárias foram: Escala de Satisfação com o Suporte Social (ESS) que mede a satisfação no geral e em quatro dimensões do suporte social - a satisfação com os amigos, a intimidade, satisfação com a família e atividades sociais - (Marôco et al., 2014); socioeconômica cultural (cidade de origem - deslocamento para realização de atividades acadêmicas-, renda, sexo, idade, dentre outras). 
Com relação a esta última, após a coleta foi observada uma potência (análise post hoc) sob a necessidade de agrupamento das cidades de origem por região de integração do estado do Pará, considerando o deslocamento para realização de atividades acadêmicas na universidade, sendo estas subdivididas por região:

\section{1- Grupo 1:}

1.1 Região do Guajará: Inclusos nessa categoria os municípios de origem: Ananindeua, Belém, Benevides, Marituba e Santa Bárbara do Pará.

1.2 Região Tocantins: Inclusos nessa categoria os municípios de origem: Abaetetuba, Acará, Baião, Barcarena, Cametá, Igarapé-Miri, Limoeiro do Ajuru, Mocajuba, Moju, Oeiras do Pará e Tailândia.

1.3 Região Rio Caeté: Inclusos nessa categoria os municípios de origem: Augusto Corrêa, Bonito; Bragança; Cachoeira do Piriá; Capanema; Nova Timboteua; Peixe-boi; Primavera; Quatipuru; Salinópolis; Santa luzia do Pará; Santarém Novo; São João de Pirabas; Tracuateua; Viseu.

1.4 Região Guamá: Inclusos nessa categoria os municípios de origem: Castanhal, Colares, Curuçá, Igarapé-Açu; Inhangapi; Magalhães Barata; Maracanã; Marapanim; Santo Antônio do Tauá; Santa Maria do Pará; Santa Izabel do Pará; São Caetano de Odivelas; São Domingos do Capim; São Francisco do Pará; São João da Ponta; São Miguel do Guamá; Terra Alta; Vigia.

\section{2- Grupo 2:}

Inclusos nessa categoria os demais municípios de origem

\section{3- Grupo 3:}

Municípios fora do Pará.

Já a Escala de Satisfação com o Suporte social (ESS) escala também utilizada na pesquisa, é composta de 15 itens na sua versão final, de autopreenchimento, onde são avaliados: satisfação com amigos; satisfação com a família; atividades sociais e intimidade. $\mathrm{O}$ instrumento não apresenta uma escala classificatória. $\mathrm{O}$ valor máximo a ser atingido é de 75 pontos, considerando que quanto maior a pontuação, maior a satisfação com o suporte social o indivíduo possui (Marôco et al., 2014).

Foram aplicados métodos estatísticos descritivos, como médias e desvios-padrão, para variáveis contínuas, frequências absolutas e relativas para variáveis dicotômicas, bem como medianas, interquartis e amplitudes para variáveis com distribuição não gaussiana.

As variáveis secundárias foram comparadas entre dois grandes grupos baseados nas variáveis primárias: a) estudantes com ansiedade (escala de Hamilton $\geq 18$ ); e b) pacientes sem ansiedade (escala de Hamilton $<18$ ), bem como entre estudantes com: c) ansiedade grave (escala de Hamilton $\geq 30$ ) e d) ansiedade não grave (escala de Hamilton $\leq 30$ ). Em função da natureza dicotômica da variável primária, as comparações foram feitas por meio de cálculos de riscos relativos, para as variáveis secundárias de natureza dicotômica ou categórica. Para variáveis contínuas de distribuição normal, foi utilizado o teste tStudent. Já em casos de distribuição não gaussiana, os grupos foram comparados, por meio do teste de Mann Whitney. Para ambos os tipos de variáveis, dicotômicas ou contínuas, as estimativas foram seguidas de seus respectivos intervalos de confiança a $95 \%$.

A pesquisa foi desenvolvida junto aos discentes devidamente matriculados nos cursos de graduação da área da saúde da Universidade do Estado do Pará na cidade de Belém, sendo estes: Biomedicina, Educação Física, Fisioterapia, Medicina, Terapia Ocupacional, no período de fevereiro a julho de 2021.

Para realização do estudo, foi composta uma amostra de 704 alunos dos cursos de graduação citados anteriormente, com idade igual ou superior a 18 anos, que foram identificados de forma aleatória estratificada, a partir de uma lista de todo 
universo disponível no momento da análise ( $\mathrm{N}=2.175)$. Tal tamanho amostral baseou-se em erro amostral de 3\% e uma proporção esperada de 58\% de alunos com transtorno de ansiedade, conforme dados da Associação Nacional dos Dirigentes da Instituições Federais de Ensino Superior (ANDIFES) (2014), apesar de ter sido detectada uma considerável variação na literatura de 36 a 77\% conforme trazida nas pesquisas de Leão et al. (2018), Vilchez-Cornejo et al (2016), Tabalipa et al. (2015) e Leal et al. (2010). Sendo assim, assumiu-se como mais adequado o percentual de 58\%, pois corresponde aproximadamente a média dos demais estudos isolados.

Os alunos de cada curso foram estratificados de acordo com o ano do curso.

Visando a adequada representatividade de cada curso e ano, na amostra como um todo, a estratificação foi proporcional ao total de alunos de cada curso individualmente, conforme indicado na Tabela 1.

Tabela 1 - Tamanho Amostral de alunos por curso UEPA (CCBS) - 2019.

\begin{tabular}{|c|c|c|c|c|}
\hline CURSO & $\begin{array}{l}\text { TOTAL DE } \\
\text { ALUNOS }\end{array}$ & PERCENTUAL & Ano & $\begin{array}{c}\text { TAMANHO AMOSTRAL } \\
(\mathbf{N})\end{array}$ \\
\hline \multirow{5}{*}{$\begin{array}{l}\text { TERAPIA } \\
\text { OCUPACIONAL }\end{array}$} & \multirow{5}{*}{167} & \multirow{5}{*}{7,68} & 1 & 11 \\
\hline & & & 2 & 11 \\
\hline & & & 3 & 11 \\
\hline & & & 4 & 11 \\
\hline & & & 5 & 11 \\
\hline \multirow{4}{*}{ BIOMEDICINA } & \multirow{4}{*}{108} & \multirow{4}{*}{4,97} & 1 & 9 \\
\hline & & & 2 & 9 \\
\hline & & & 3 & 9 \\
\hline & & & 4 & 9 \\
\hline \multirow{6}{*}{ MEDICINA } & \multirow{6}{*}{608} & \multirow{6}{*}{27,95} & 1 & 33 \\
\hline & & & 2 & 33 \\
\hline & & & 3 & 33 \\
\hline & & & 4 & 33 \\
\hline & & & 5 & 33 \\
\hline & & & 6 & 33 \\
\hline \multirow{5}{*}{ FISIOTERAPIA } & \multirow{5}{*}{153} & \multirow{5}{*}{7,03} & 1 & 10 \\
\hline & & & 2 & 10 \\
\hline & & & 3 & 10 \\
\hline & & & 4 & 10 \\
\hline & & & 5 & 10 \\
\hline \multirow{4}{*}{ EDUCAÇÃO FÍSICA } & \multirow{4}{*}{653} & \multirow{4}{*}{30,02} & 1 & 53 \\
\hline & & & 2 & 53 \\
\hline & & & 3 & 53 \\
\hline & & & 4 & 53 \\
\hline
\end{tabular}




\begin{tabular}{|c|c|c|c|c|}
\hline \multirow{5}{*}{ ENFERMAGEM } & \multirow{5}{*}{486} & \multirow{5}{*}{22,34} & 1 & 31 \\
\hline & & & 2 & 31 \\
\hline & & & 3 & 31 \\
\hline & & & 4 & 31 \\
\hline & & & 5 & 31 \\
\hline TOTAL GERAL & 2175 & & & 706 \\
\hline
\end{tabular}

Fonte: Coordenação de Registro e Controle Acadêmico (2019).

\section{Resultados}

Quanto aos resultados obtidos na pesquisa, inicialmente, foram reportadas as médias dos escores de Hamilton obtidos nos cursos avaliados: Biomedicina, Educação Física, Fisioterapia, Medicina e Terapia Ocupacional, tendo sido observadas as médias de 17,1, 17,7, 16,8,15,5, 17,7, respectivamente. Assim, o curso com média mais próxima ao escore de ansiedade leve (maior ou igual a 18), foram os cursos de Educação Física e Terapia Ocupacional, conforme a Tabela 2.

Tabela 2- Média de escore de Hamilton por cursos.

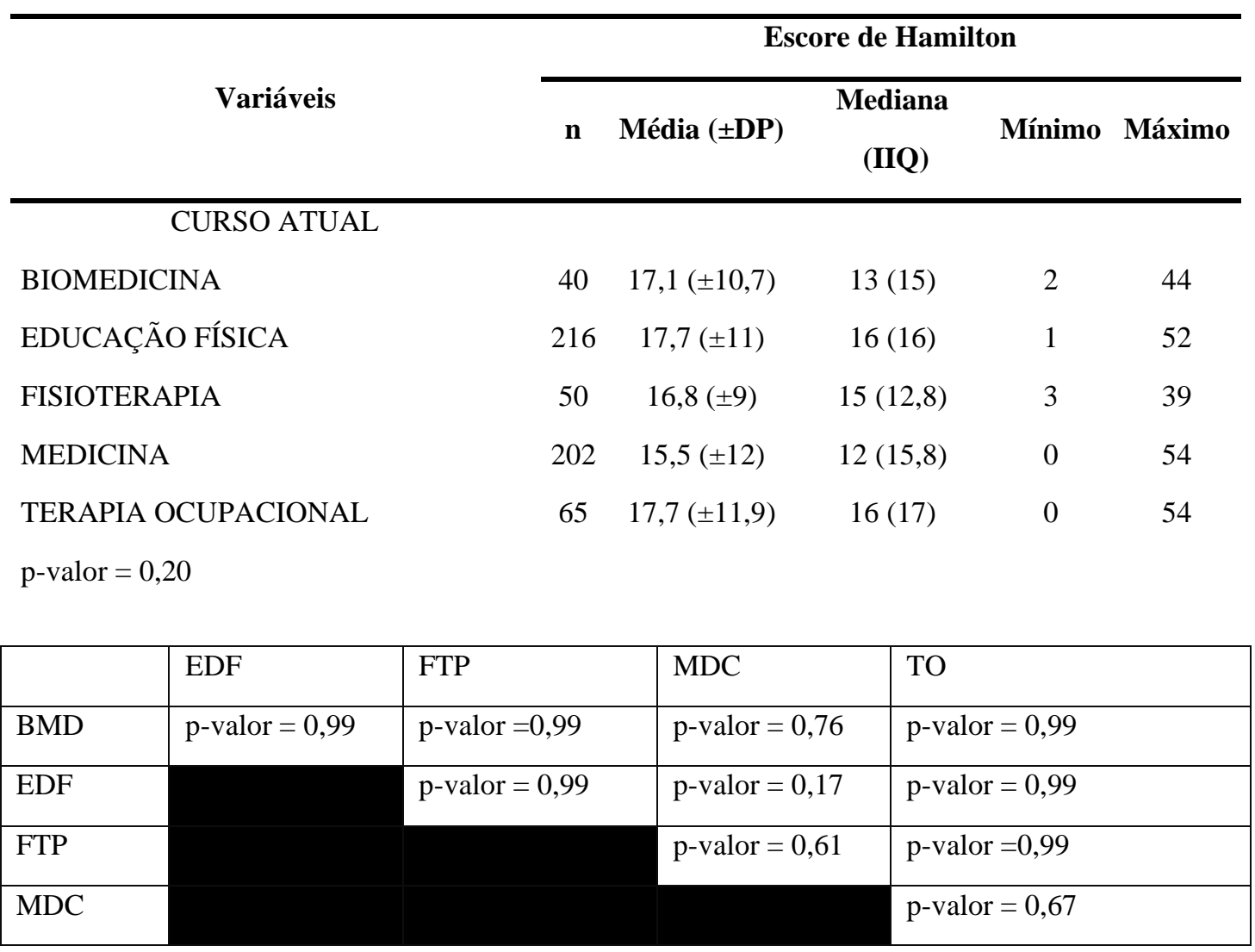

Valores de $\mathrm{p}$ (Teste de Post Hoc Dwass-Steel), nas comparações de 2x2.

BMD, biomedicina; EDF, educação física; FTP, fisioterapia; MDC, medicina; TO, terapia ocupacional P>0,05 para todas as comparações. Fonte: Pesquisa de Campo (2021).

Já na relação entre o ano o qual os acadêmicos encontram-se matriculados e o escore de Hamilton, pode-se perceber que a média do escore de Hamilton apresentada foi maior em discentes cursando o $3^{\circ}$ e o $2^{\circ}$ anos, segundo os valores médios 
de 18,2 e 18, respectivamente, revelando escores relacionados a ansiedade leve, seguido por acadêmicos do $5^{\circ}$ ano (média de 16,3); do $1^{\circ}$ ano (média de 16); $6^{\circ}$ ano (média de 15,9), $4^{\circ}$ ano (média de 15,6), conforme ilustrado pela Tabela 3.

Tabela 3 - Ano o qual está cursando x escore de Hamilton.

\begin{tabular}{|c|c|c|c|c|c|}
\hline \multirow[b]{2}{*}{ Variáveis } & \multicolumn{5}{|c|}{ Escore de Hamilton } \\
\hline & $\mathbf{n}$ & $\begin{array}{l}\text { Média } \\
( \pm \mathrm{DP})\end{array}$ & $\begin{array}{c}\text { Mediana } \\
\text { (IIQ) }\end{array}$ & Mínimo & Máximo \\
\hline \multicolumn{6}{|c|}{ ANO O QUAL ESTÁ CURSANDO } \\
\hline & $\mathrm{N}$ & Média (DP) & Mediana & Mínimo & Máximo \\
\hline $1^{\circ} \mathrm{ANO}$ & 118 & $16( \pm 10,7)$ & $13(16)$ & 0 & 54 \\
\hline $2^{\circ} \mathrm{ANO}$ & 117 & $18( \pm 10,9)$ & $16(14)$ & 0 & 54 \\
\hline $3^{\circ} \mathrm{ANO}$ & 117 & $18,2( \pm 11,5)$ & $16(18)$ & 1 & 52 \\
\hline $4^{\circ} \mathrm{ANO}$ & 125 & $15,6( \pm 9,8)$ & $13(13)$ & 1 & 51 \\
\hline $5^{\circ} \mathrm{ANO}$ & 62 & $16,3( \pm 11)$ & $15(15,8)$ & 0 & 49 \\
\hline $6^{\circ} \mathrm{ANO}$ & 34 & $15,9( \pm 11,70)$ & $10,5(15,5)$ & 2 & 44 \\
\hline
\end{tabular}

DP = Desvio-padrão, IIQ=Intervalo Interquartil, *Teste de Kruskal-Wallis

\begin{tabular}{|l|l|l|l|l|l|}
\hline & $2^{\text {o }}$ & $3^{\text {o }}$ & $4^{\text {o }}$ & $5^{\text {o }}$ & $6^{\text {o }}$ \\
\hline $1^{\text {o }}$ & p-valor $=0,60$ & p-valor $=0,66$ & p-valor $=0,99$ & p-valor $=0,99$ & p-valor $=0,99$ \\
\hline $2^{\mathbf{o}}$ & & p-valor $=0,99$ & p-valor $=0,51$ & p-valor $=0,85$ & p-valor $=0,71$ \\
\hline $3^{\text {o }}$ & & p-valor $=0,62$ & p-valor $=0,89$ & p-valor $=0,86$ \\
\hline $4^{\mathbf{o}}$ & & & p-valor $=0,99$ & p-valor $=0,99$ \\
\hline $5^{\text {o }}$ & & & & p-valor $=0,99$ \\
\hline
\end{tabular}

P>0,05 para todas as comparações. Fonte: Pesquisa de Campo (2021).

No que se refere à condição socioeconômica e às rendas individuais mensais, as mesmas foram divididas por grupos. O primeiro, de R\$ 998 reais (grupo I); entre R\$ 998 e R\$ 1995 (grupo II); entre R\$ 1996 e R\$ 2993 (grupo III); entre R\$ 2994 e R\$ 3991 (grupo IV); entre R\$ 3992 e R\$ 4989 (grupo V) entre R\$ 4990 e R\$ 5987 (grupo VI) entre R\$ 5988 e R\$ 6985 (grupo VII); entre R\$ 6986 e R \$ 7983 (grupo VIII); entre R\$ 7984 e R\$ 8981 (grupo IX); mais de 10 salários mínimos (grupo $\mathrm{X})$.

Quando realizado o teste de associação entre a renda individual e o escore de Hamilton, através da média, observouse que o grupo com menor renda (grupo I) apresentou maior média de escore de Hamilton para transtorno de ansiedade: 17,4 com média mais próxima ao escore de ansiedade leve (maior ou igual a 18); grupo II: média de 16,4; grupo III: média de 14,7; grupo IV: média de 9,11; grupo V: média de 7; grupo VI: média de 14; grupo VII: média de 3; grupo IX: média 3, conforme mostra Tabela 4. 
Tabela 4 - Renda mensal x Escore de Hamilton (continua).

\begin{tabular}{llcccc}
\hline & \multicolumn{4}{c}{ Escore de Hamilton } \\
\cline { 3 - 5 } Variáveis & n & Média $( \pm D P)$ & Mediana & Mínimo & Máximo \\
& & & & (IIQ) & \\
\hline
\end{tabular}

RENDA INDIVIDUAL (MÊS)

ATÉ R\$ 998 (GRUPO I)

ENTRE R\$ 998 E R\$ 1995 (GRUPO II)

ENTRE R\$ 1996 E R\$ 2993 (GRUPO III)

ENTRE R\$ 2994 E R\$ 3991 (GRUPO IV)

ENTRE R\$ 3992 E R\$ 4989 (GRUPO V)

ENTRE R\$ 4990 E R\$ 5987 (GRUPO VI)

ENTRE R\$ 5988 E R \$ 6985 (GRUPO VII)

ENTRE R\$ 6986 E R\$ 7983 (GRUPO VIII) 1

ENTRE R\$ 7984 E R\$ 8981 (GRUPO IX)

$>10$ SM (GRUPO X)

p-valor $=0,011($ Kruskal-Wallis $)$

$\begin{array}{ccccc}442 & 17,4( \pm 10,9) & 15(16) & 0 & 54 \\ 84 & 16,4( \pm 10,9) & 12,5(16,3) & 1 & 49 \\ 19 & 14,7( \pm 11,4) & 11(12,5) & 2 & 52 \\ 9 & 9,11( \pm 4,5) & 10(6) & 4 & 18 \\ 4 & 7( \pm 5,1) & 8(4,5) & 0 & 12 \\ 7 & 14( \pm 8,1) & 11(10) & 5 & 28 \\ 3 & 13,7( \pm 10,7) & 8(9,5) & 7 & 26 \\ 1 & - & - & - & - \\ 3 & 4,33( \pm 2,3) & 3(2) & 3 & 7 \\ 1 & - & - & - & -\end{array}$

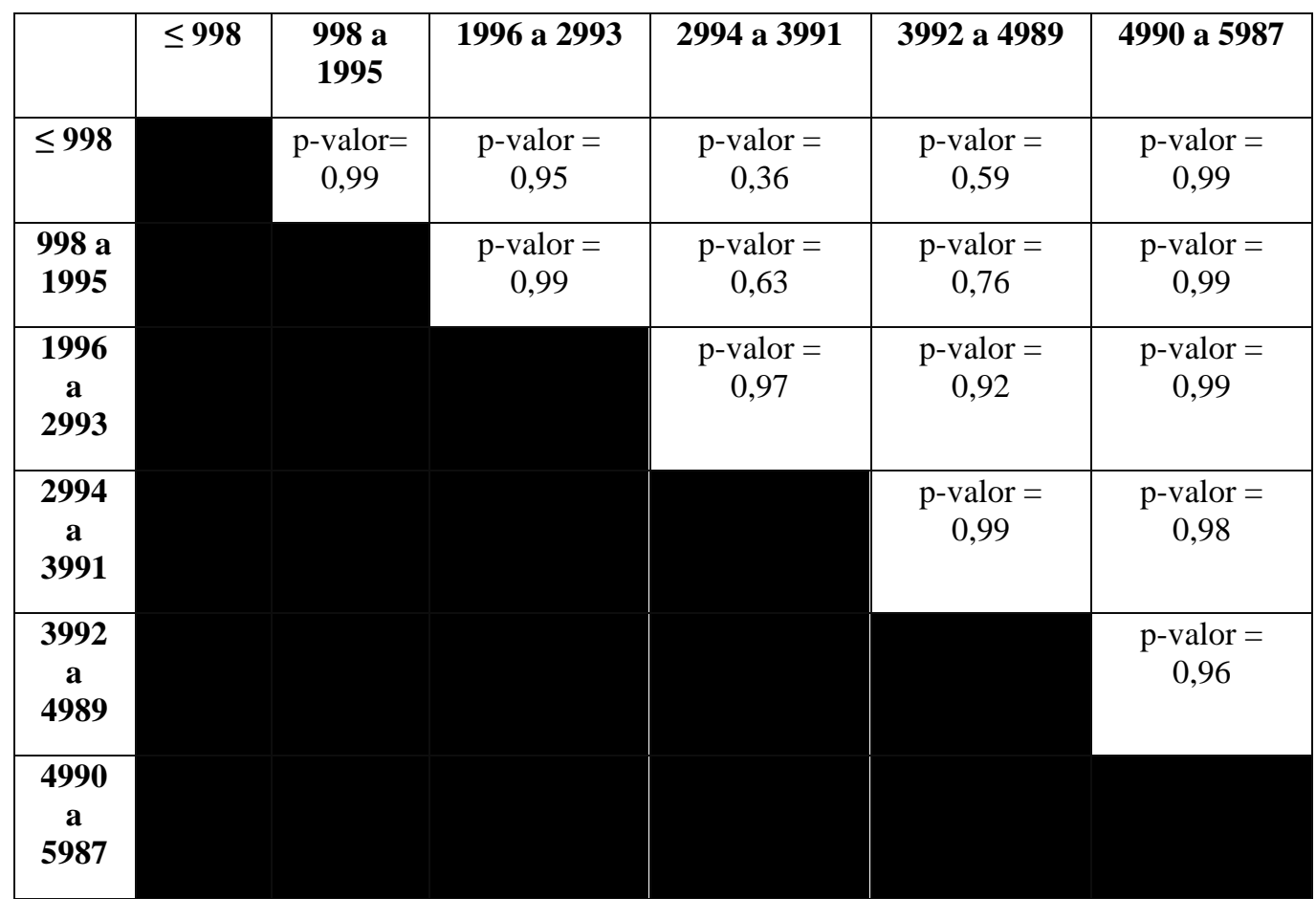

Teste de Post Hoc Dwass-Steel

Erros do Tipo I (p) para as comparações entre todos os pares de faixas renda expressas em moeda nacional do Brasil (Reais). $\mathrm{P}>0,05$ para todas as comparações. Fonte: Pesquisa de Campo (2021). 
Com relação a diferença entre a média do escore de Hamilton e o sexo dos discentes, observou-se maior escore entre o sexo feminino: média de 19,8, configurando ansiedade leve, maior que 18. Já entre o sexo masculino, a média do escore de Hamilton foi de 14,2, como mostra a Tabela 5.

Tabela 5- Sexo x Escore de Hamilton.

\begin{tabular}{|c|c|c|c|c|c|}
\hline \multirow[b]{2}{*}{ Variáveis } & \multicolumn{5}{|c|}{ Escore de Hamilton } \\
\hline & $\mathbf{n}$ & Média $( \pm D P)$ & $\begin{array}{c}\text { Mediana } \\
\text { (IIQ) }\end{array}$ & Mínimo & Máximo \\
\hline \multicolumn{6}{|l|}{ SEXO } \\
\hline MASCULINO & 304 & $14,2( \pm 9,2)$ & $12(13)$ & 0 & 52 \\
\hline FEMININO & 269 & $19,8( \pm 11,8)$ & $18(18)$ & 1 & 54 \\
\hline
\end{tabular}

Fonte: Pesquisa de Campo (2021).

Tabela 6 - Região de origem (por região de integração) x Escore de Hamilton.

\begin{tabular}{|c|c|c|c|c|c|}
\hline \multirow[b]{2}{*}{ Variáveis } & \multicolumn{5}{|c|}{ Escore de Hamilton } \\
\hline & $\mathbf{n}$ & Média $( \pm \mathrm{DP})$ & $\begin{array}{c}\text { Mediana } \\
\text { (IIQ) }\end{array}$ & Mínimo & Máximo \\
\hline REGIÃO DE INTEGRAÇÃO & & & & & \\
\hline Região do Guajará* & 456 & $\begin{array}{c}16,9 \\
( \pm 10,9)\end{array}$ & $14,5(16)$ & 0 & 54 \\
\hline Região Guamá* & 26 & $\begin{array}{c}17,3 \\
( \pm 11,4)\end{array}$ & $13(19,8)$ & 2 & 39 \\
\hline Região do Tocantins* & 22 & $\begin{array}{c}16,5 \\
( \pm 12,1)\end{array}$ & $12(16,8)$ & 2 & 42 \\
\hline Região Rio Caeté** & 13 & $12,3( \pm 6,8)$ & $12(8)$ & 3 & 25 \\
\hline Demais municípios do Pará*** & 28 & $17,5( \pm 9,9)$ & $15(16,8)$ & 5 & 36 \\
\hline $\begin{array}{l}\text { Fora do Pará*** } \\
\text { p-valor }=0,79 \text { (Kruskal-Wallis) }\end{array}$ & 28 & $\begin{array}{c}15,8 \\
( \pm 10,3)\end{array}$ & $14(14,8)$ & 0 & 43 \\
\hline
\end{tabular}

No que se refere ao município de origem dos acadêmicos, considerando a distância para realizações de suas atividades acadêmicas e a relação com os escores de Hamilton para o transtorno de ansiedade, observou-se que a média com os maiores escores foram apresentadas pelos acadêmicos oriundos dos demais municípios do Pará, ou seja, os mais distantes, com média de 17,5 no escore, o mais próximo dos transtornos de ansiedade leve. Seguido pelos discentes advindos das regiões: Guamá (média 17,3); Guajará (média 16,9); Tocantins (média 16,5); Fora do Pará (média 15,8); Caeté (média 12,3). Conforme ilustrado pela Tabela 6 . 


\begin{tabular}{|l|l|l|l|l|l|l|}
\hline & $\begin{array}{l}\text { Região do } \\
\text { Guajará }\end{array}$ & Região Guamá & $\begin{array}{l}\text { Região do } \\
\text { Tocantins }\end{array}$ & $\begin{array}{l}\text { Região Rio } \\
\text { Caeté }\end{array}$ & $\begin{array}{l}\text { Demais } \\
\text { municípios do } \\
\text { Pará }\end{array}$ & Fora do Pará \\
\hline $\begin{array}{l}\text { Região do } \\
\text { Guajará }\end{array}$ & & p-valor=0,99 & p-valor $=0,99$ & p-valor $=0,77$ & p-valor $=0,99$ & p-valor $=0,99$ \\
\hline $\begin{array}{l}\text { Região } \\
\text { Guamá }\end{array}$ & & p-valor $=0,99$ & p-valor $=0,86$ & p-valor $=0,99$ & p-valor $=0,99$ \\
\hline $\begin{array}{l}\text { Região do } \\
\text { Tocantins }\end{array}$ & & & p-valor $=0,99$ & p-valor $=0,99$ & p-valor $=0,99$ \\
\hline $\begin{array}{l}\text { Região } \\
\text { Rio Caeté }\end{array}$ & & & & p-valor $=0,70$ & -valor $=0,96$ \\
\hline $\begin{array}{l}\text { Demais } \\
\text { municípios } \\
\text { do Pará }\end{array}$ & & & & & & p-valor $=0,98$ \\
\hline $\begin{array}{l}\text { Demais } \\
\text { municípios } \\
\text { do Pará }\end{array}$
\end{tabular}

Teste de Post Hoc Dwass-Steel

$\mathrm{P}>0,05$ para todas as comparações.

Regiões de integração e demais municípios ordenados de forma crescente em relação à distância do município de origem à instituição de ensino. *, regiões consideradas adjacentes; **, moderadamente distantes e $* * *$, mais distantes.

Fonte: Pesquisa de Campo (2021).

No que tange as variáveis de suporte social (que fazem parte da Escala de Suporte Social) quando associadas à Escala de Hamilton, na Tabela 8, observou-se que as médias dos escores apresentados relacionavam-se às variáveis referentes à falta de suporte social recebido. Como mostrado na variável 1: "Por vezes sinto-me só no mundo e sem apoio", com média no escore de Hamilton de 22,4, classificado como ansiedade leve, enquanto os que não se sentem dessa forma apresentaram uma média de escore de 14,1, ou seja, sem ansiedade. O mesmo ocorre com a variável 2: "Não saio com amigos tantas vezes quanto eu gostaria", com média no escore de Hamilton de 18,1, classificado como ansiedade leve, enquanto os que estão satisfeitos apresentaram uma média de escore de 14,6, ou seja, sem ansiedade. Com relação a variável 3 "Os amigos não me procuram tantas vezes quanto eu gostaria", a média no escore de Hamilton de 20,3, classificado como ansiedade leve, enquanto os outros apresentaram uma média de escore de 14,6, ou seja, sem ansiedade.

A variável 4 "Quando preciso desabafar com alguém não encontro facilmente amigos com quem o fazer" demonstrou uma média de 19,9, ansiedade leve, no escore de Hamilton de indivíduos que não encontram essa rede, já os que encontram, uma média de 14,5 no escore de Hamilton (sem ansiedade). Quanto a variável 5 "Mesmo nas situações mais embaraçosas, se precisar de apoio de emergência não tenho várias pessoas a quem posso recorrer”, a média no escore de Hamilton de indivíduos que não tem as pessoas com quem recorrer foi de 20,2 (ansiedade leve), já as que possuem essas pessoas com as quais possam recorrer apresentaram média no escore de 15 (sem ansiedade).

A variável 6 "Às vezes sinto falta de alguém verdadeiramente íntimo que me compreenda e com quem possa desabafar sobre coisas íntimas", os indivíduos que confirmaram essa afirmativa apresentaram média de escore de ansiedade de 19,9 (ansiedade leve), os que negaram, apresentaram escore de 14,8 (sem ansiedade). Quanto a variável 7 "Sinto falta de atividades sociais que me satisfaçam" os indivíduos que sentem falta dessas atividades sociais apresentaram média de escore de 18,6 (ansiedade leve), os que não sentem falta tiveram média de escore de 12,9 (sem ansiedade).

A variável 8 "Gostaria de participar mais em atividades de organizações (p.ex. clubes desportivos, escuteiros, partidos políticos, etc.)" revela que os indivíduos que gostariam de participar mais apresentaram média de escore de 18,2 (ansiedade leve), e os casos negativos 15,4 (sem ansiedade). A variável 9 "Estou insatisfeito com a forma como me relaciono com a minha família", mostra que os acadêmicos insatisfeitos tiveram média de escore de ansiedade de 20,6 (ansiedade leve), já os participantes que responderam estarem satisfeitos, apresentaram escore de 15,5 (sem ansiedade). 
Quanto à satisfação relacionada ao tempo que os participantes passam com familiares, revelada na variável 10 "Estou insatisfeito com a quantidade de tempo que passo com a minha família”, os acadêmicos que se disseram insatisfeitos tiveram escore de 20,6 (ansiedade leve), enquanto os que não se encontravam insatisfeitos apresentaram escore de 15,3 (sem ansiedade). A variável 11 "Estou insatisfeito com o que faço em conjunto com a minha família" revela que os discentes que se encontravam insatisfeitos tiveram média de escore de Hamilton para transtorno de ansiedade de 20,7 (ansiedade leve) e 15,3 (sem ansiedade) dos que demonstraram estarem satisfeitos.

A variável 12 "Estou insatisfeito com a quantidade de amigos que tenho", mostra que a média do escore de Hamilton dos acadêmicos que se encontravam insatisfeitos foi de 21,9 (ansiedade leve), os que não estão insatisfeitos tiveram escore de 15,9 (sem ansiedade). Na variável 13 "Estou insatisfeito com a quantidade de tempo que passo com os meus amigos", a média do escore de Hamilton entre os que concordaram com a afirmativa foi de 19 (ansiedade leve), já os que discordaram da afirmativa tiveram média de 14,4 (sem ansiedade).

Com relação a variável 14 "Estou insatisfeito com as atividades e coisas que faço com o meu grupo de amigos", a média do escore de ansiedade dos acadêmicos insatisfeitos foi de 19,7 (ansiedade leve), e os que não demonstraram insatisfação tiveram escore de 15,3 (sem ansiedade). Na última variável da escala, a vaiável 15, "Estou insatisfeito com o tipo de amigos que tenho", a média no escore de ansiedade dos que se encontravam insatisfeitos foi de 19,2 (ansiedade leve), enquanto os que se diziam satisfeitos tiveram média no escore de 16,6 (sem ansiedade). Essas informações podem ser visualizadas na Tabela 7.

Tabela 7 - Escala de Suporte Social x Escore de Hamilton (continua).

\begin{tabular}{llllll}
\hline & \multicolumn{4}{c}{7} & \\
\hline & Variáveis & n & Média $( \pm D P)$ & $\begin{array}{c}\text { Mediana } \\
\text { (IIQ) }\end{array}$ & Mínimo Máximo \\
\cline { 2 - 5 } & & &
\end{tabular}

1-Por vezes sinto-me só no mundo e sem apoio

Não

Sim

384

$14,1( \pm 9,5)$

$11,0(12,3)$

0

52

$18922,4( \pm 11,2)$

$22(16)$

54

2-Não saio com amigos tantas vezes quanto eu gostaria

Não

$208 \quad 14,6( \pm 10,6)$

$36518,1( \pm 10,8)$

$11(14)$

$16(16)$

52

Sim

3-Os amigos não me procuram tantas vezes quanto eu gostaria

Não

$348 \quad 14,6( \pm 9,9)$

$12(14)$

19 (16)

52

Sim

$22520,3( \pm 11,2)$

$19(16)$

4-Quando preciso desabafar com alguém não encontro facilmente amigos com quem o fazer

Não

Sim

$328 \quad 14,5( \pm 10,5)$

$11(13)$

5-Mesmo nas situações mais embaraçosas, se precisar de apoio de emergência não tenho várias pessoas a quem posso recorrer Não 
6-Às vezes sinto falta de alguém verdadeiramente íntimo que me compreenda e com quem possa desabafar sobre coisas íntimas

Não

$347 \quad 14,8( \pm 10,3)$

$12(14)$

51

Sim

7-Sinto falta de atividades sociais que me satisfaçam

Não

$17912,9( \pm 9,8) \quad 10(11,5) \quad 0 \quad 52$

Sim

$39418,6( \pm 10,8) \quad 17(15) \quad 114$

8-Gostaria de participar mais em atividades de organizações (p.ex. clubes desportivos, escuteiros,partidos políticos, etc.)

Não

Sim

9-Estou insatisfeito com a forma como me relaciono com a minha família

Não

Sim

10-Estou insatisfeito com a quantidade de tempo que passo com a minha família

Não

Sim

11-Estou insatisfeito com o que faço em conjunto com a minha família

Não

Sim

$413 \quad 15,3( \pm 10,2)$

$160 \quad 20,7( \pm 11,4)$

$12(15)$

$19,5(17)$

51

$431 \quad 15,5( \pm 10,5)$

$12(14)$

$12(15)$

16 (16)

46

54

$410 \quad 15,3( \pm 10,4) \quad 12(15) \quad 0 \quad 52$

$16320,6( \pm 11,1) \quad 20(15,5) \quad 2 \quad 54$

12-Estou insatisfeito com a quantidade de amigos que tenho

Não

Sim

$48515,9( \pm 10,5)$

$12(15)$

$21(15)$

52

$88 \quad 21,9( \pm 11,3) \quad 21(15) \quad 3 \quad 54$

13-Estou insatisfeito com a quantidade de tempo que passo com os meus amigos

Não

$270 \quad 14,4( \pm 10,7) \quad 11(13)$

$303 \quad 19( \pm 10,5) \quad 17(16) \quad 0054$

54

14-Estou insatisfeito com as atividades e coisas que faço com o meu grupo de amigos Não

Sim

$381 \quad 15,3( \pm 10,2)$

$19219,7( \pm 11,4)$

$12(15)$

$17(15,3)$

51

15-Estou insatisfeito com o tipo de amigos que tenho

Não

Sim
$522 \quad 16,6( \pm 10,7)$

$51 \quad 19,2( \pm 11,7)$
14 (16)

17 (14)

4




\section{Discussão}

Os resultados da pesquisa demonstraram prevalência dos transtornos de ansiedade nos primeiros anos de curso, com relação aos acadêmicos do segundo e terceiro anos de curso, pode-se perceber que a média do escore de Hamilton apresentada foi maior nestes grupos de discentes, com valores médios de 18 e 18,2 respectivamente, revelando escores relacionados a ansiedade leve.

Esta informação também foi observada nos estudos de Grether et al (2019), que ao avaliar a prevalência de transtornos mentais comuns (TMC) entre 340 alunos do primeiro ao décimo segundo semestre do curso de Medicina no ano de 2017 da Universidade Regional de Blumenau, constatou que a prevalência dos TMC foi maior nos dois primeiros anos do curso, tendo a menor prevalência nos dois últimos anos da faculdade.

Observaram-se os mesmos resultados na pesquisa de Costa et al (2020) com 279 estudantes de medicina da Universidade Federal do Rio Grande do Norte em que demonstrou que os estudantes que apresentaram graus mais elevados de sintomatologia ansiosa estavam nos primeiros dois ciclos do curso.

Os acadêmicos nos primeiros anos de curso demonstraram maiores riscos para desenvolverem transtornos de ansiedade, o início da graduação pode ser de fato o momento mais complicado de se enfrentar. Nos primeiros anos de graduação, com as mudanças advindas com as novas responsabilidades, novos costumes, regras e dificuldades de adaptação podem estar associados ao aumento destes riscos (Costa et al 2017).

No teste de associação entre a renda individual e o escore de Hamilton, através da média, observou-se que o grupo com menor renda, de até $\mathrm{R} \$ 998,00$, apresentou maior média de escore de Hamilton para transtorno de ansiedade: 17,4 com média mais próxima ao escore de ansiedade leve (maior ou igual a 18).

Esta evidência também foi encontrada nos estudos de Costa et al (2019), que ao realizar um estudo transversal de base populacional com 1.953 indivíduos entre 18 e 35 anos, considerando avaliação das variáveis sociodemográfica e os transtornos de ansiedade, observou que problemas econômicos também podem estar relacionados à alta prevalência desses quadros, em virtude da dificuldade de cumprir com a responsabilidade financeiras, comprar bens necessários e manter bons hábitos de vida.

A pesquisa de Melo et al (2020) ao avaliar o perfil epidemiológico de 101 pacientes com transtorno de ansiedade assistidos pelo CAPS II no município de Santarém, no período de 2016 e 2017, corrobora com o resultado encontrado, no qual cita que maioria dos pacientes vive com até meio salário mínimo.

Com relação a diferença entre a média do escore de Hamilton e o sexo dos discentes, observou-se maior escore entre o sexo feminino: média de 19,8, configurando ansiedade leve, maior que 18. Já entre o sexo masculino, a média do escore de Hamilton foi de 14,2, ou seja, sem ansiedade. Os Estudos de Leão et al (2018), realizado com 476 estudantes universitários da área da saúde, que tinham como objetivo estimar a prevalência e os fatores associados à depressão e ansiedade em estudantes universitários da área da saúde, revelam que a prevalência de ansiedade esteve mais associada ao sexo feminino.

Estas evidências são mostradas também na pesquisa de Cruz et al (2020), na qual a variável sexo, mostrou significativa diferença entre homens e mulheres, onde o sexo feminino apresentou maior percentual de indivíduos com ansiedade em nível normal e leve.

Um dos fatores que está associada a essa prevalência é o fato de que a sociedade ainda possui dificuldades para as mulheres adquirirem autonomia social e financeira, submetendo-as a maiores conflitos, inseguranças, provocando uma reação de maior ansiedade diante de situações de pressão psicológica (Carvalho et al 2015).

Quanto ao deslocamento dos discentes de seu município de origem para realizações de suas atividades acadêmicas e a relação com os escores de Hamilton para o transtorno de ansiedade, observou-se que a média com os maiores escores foram apresentadas pelos acadêmicos oriundos dos demais municípios do Pará, ou seja, os mais distantes, com média de 17,5 no 
escore, o mais próximo dos transtornos de ansiedade leve. O estudo de Vizzotto et al (2017) que corrobora com essa informação foi realizado com 238 participantes, onde observou-se que as taxas de ansiedade são aumentadas em pessoas que se deslocam para outras cidades com a finalidade de estudar.

Observa-se que indivíduos que deixam suas localidades para estudar fora geralmente apresentam risco elevado para desenvolver quadros de ansiedade (Guimarães, 2014). Cardozo et al. (2016) infere que o estudante pode se sentir inseguro ao iniciar uma nova jornada na universidade, sentir que não está preparado, o que pode acabar agravando os sintomas da ansiedade, sendo então, o fato de não morar na sua cidade de origem um importante fator de risco, enfrentando maior dificuldade para criar novos vínculos, estabelecimento de um novo lar ou até mesmo medo do desconhecido.

Ao avaliar as variáveis de suporte social (que fazem parte da Escala de Suporte Social) quando associadas à Escala de Hamilton, observou-se que as médias dos escores apresentados relacionavam-se às variáveis referentes à falta de suporte social recebido. Ou seja, para todas as variáveis da escala de suporte social, que determinavam a falta do suporte recebido pelos estudantes com relação à família, amigos, pessoas com quem possa contar, atividades sociais às quais participa, pessoas que lhes ofereçam apoio, tempo livre com os amigos, pessoas com quem desabafar, participação social e em comunidade, os escores de ansiedade manifestavam ansiedade leve.

Esses resultados observados reforçam a ideia trazida nos estudos de Souza (2017), no qual foram avaliados 384 estudantes da Universidade Federal do Triângulo Mineiro (UFTM) e mostraram que 47\% dos estudantes revelaram sentimento de solidão em algum nível, nos estudantes investigados foi possível identificar uma parcela que revelavam déficits em seu suporte social.

A percepção do suporte conforme afirma Oliveira \& Dias (2014), é essencial para auxiliar no processo formador dos estudantes no contexto universitário, sendo identificado como fator importante para ampliação da rede de apoio ao longo da graduação.

Outra evidência que consolidam os indícios observados foram os estudos de Oliveira \& Barroso (2020) no qual realizou-se junto a 48 universitários do curso de Psicologia da Universidade Federal do Triângulo Mineiro que avaliaram níveis de solidão, sintomas depressivos e suporte social, revelaram que e 39,6\% estavam abaixo da média de Suporte Social.

\section{Conclusão}

O ambiente da Universidade exige uma série de mudanças na vida dos acadêmicos, por representar um ambiente novo, que constantemente impõe desafios aos discentes, essencialmente quando relacionado aos estudantes de cursos da área da saúde, que para além destes fatores, assumem a responsabilidade para com a vida do outro.

Esta pesquisa realizada no ambiente de uma Universidade pública estadual, junto aos acadêmicos dos cursos da área da saúde, mostrou a prevalência dos transtornos de ansiedade nos alunos que cursavam os primeiros anos dos seus respectivos cursos, revelou também prevalência dos transtornos de ansiedade no sexo feminino, conforme mostrados em outros estudos realizados anteriormente.

Outra evidência constatada com o estudo relaciona-se a prevalência dos transtornos de ansiedade dentre os alunos que vivem com menor renda individual, assim como, uma alta prevalência dos transtornos de ansiedade junto aos acadêmicos que tiveram que se deslocar de suas cidades natal para cursar a Universidade.

Uma importante evidência encontrada diz respeito a alta prevalência dos transtornos de ansiedade nos acadêmicos que relataram suporte social recebido insuficiente, seja ele da família, de amigos, assim como relativo à sua participação social, frequentando pouco ou não frequentando espaços e atividades sociais fora da Universidade. 
É imprescindível que os discentes e o corpo docente reconheçam os principais sinais de alerta relacionadas às manifestações clínicas dos transtornos de ansiedade, bem como seja qualificado para oferecer ou buscar o melhor direcionamento do acadêmico a fim de prevenir os agravos associados a esta patologia.

Portanto, ressalta-se que a pesquisa trouxe informações relevantes, relacionados a prevalência dos transtornos de ansiedade nos cursos da área da saúde, bem como pôde revelar as principais associações que colaboram para este quadro.

É importante salientar a necessidade de outros estudos utilizando as mesmas variáveis, visando investigar a relação entre a prevalência dos transtornos de ansiedade e suas possíveis associações, sendo realizado em outras instituições a fim de verificar se os resultados encontrados se assemelhariam, no que se relaciona as associações encontradas. Reforça-se, então, a importância da replicação do estudo, tanto a nível local, regional ou internacional.

\section{Referências}

American Occupational Therapy Association [AOTA] (2015). Estrutura da prática da terapia ocupacional: domínio \& processo. revista de terapia ocupacional da universidade de São Paulo, 26(3), 1-49.

American Psychiatric Association [APA] (2014). Manual diagnóstico e estatístico de transtornos mentais [recurso eletrônico]. DSM-5. Porto Alegre: Artmed, (5), 1-917.

Aragão, J. (2013). Introdução aos estudos quantitativos utilizados em pesquisas científicas. Revista Práxis, 3(6). https://doi.org/10.25119/praxis-3-6-566

Associação Nacional dos Dirigentes Das Instituições Federais De Ensino Superior [ANDIFES] (2014). IV Pesquisa do perfil sócioeconômico e cultural dos estudantes de graduação das instituições federais de ensino superior brasileiras. https://www.andifes.org.br/wp-content/uploads/2017/11/Pesquisa-de-Perfildos-Graduanso-das-IFES_2014.pdf.

Cardozo, M. Q., Gomes, K. M., Fan, L. G., \& Soratto, M. T. (2016). Fatores Associados à Ocorrência de Ansiedade dos Acadêmicos de Biomedicina. Saúde e Pesquisa, 9(2), 251. https://doi.org/10.17765/1983-1870.2016v9n2p251-262

Carvalho, E. A. d., Bertolini, S. M. M. G., Milani, R. G., \& Martins, M. C. (2015). Índice de ansiedade em universitários ingressantes e concluintes de uma instituição de ensino superior/Anxiety scores in university entering and graduating students from a higher education institution. Ciência, Cuidado $e$ Saúde, 14(3), 1290. https://doi.org/10.4025/cienccuidsaude.v14i3.23594

Costa KMV, Sousa KRS, Formiga PA, Silva WS, Bezerra EBN (Junho, 2017). Ansiedade em universitários na área da saúde. Anais do 2 Congresso Brasileiro de Ciências da Saúde, Campina Grande, PB, Brasil., 2. p. 1-10.

Costa, C. O. d., Branco, J. C., Vieira, I. S., Souza, L. D. d. M., \& Silva, R. A. d. (2019). Prevalência de ansiedade e fatores associados em adultos. Jornal Brasileiro de Psiquiatria, 68(2), 92-100. https://doi.org/10.1590/0047-2085000000232

Costa, D. S. d., Medeiros, N. d. S. B., Cordeiro, R. A., Frutuoso, E. d. S., Lopes, J. M., \& Moreira, S. d. N. T. (2020). Sin tomas de Depressão, Ansiedade e Estresse em Estudantes de Medicina e Estratégias Institucionais de Enfrentamento. Revista Brasileira de Educação Médica, 44(1). https://doi.org/10.1590/1981-5271v44.1-20190069

Cruz, M. C. N. L., Gonçalves, F. T. D., Melo, K. C., Soares, A. N., Silva, W. C. d., Silva, C. O. d., Mesquita, Z. A., Gonçalves, F. T. D., Araújo, B. F., Costa, E. M., Neto, A. H. d. N., Oliveira, M. C. d. S., Rodrigues, R. P. D., Chagas, A. F. d., \& Ibiapina, C. C. (2020). Ansiedade em universitários iniciantes de cursos da área da saúde / Anxiety in university beginners of health courses. Brazilian Journal of Health Review, 3(5), 14644-14662. https://doi.org/10.34119/bjhrv3n5-259.

Gerhardt, t., \& Silveira, d. (Orgs.). (2009). Métodos de pesquisa. Editora da UFRGS. https://lume.ufrgs.br/handle/10183/52806

Gomes, C., Araújo, C. L., \& Comonian, J. O. (2018). Sofrimento psíquico na Universidade: uma análise dos sentidos configurados por acadêmicos. Revista Psicologia, Diversidade e Saúde, 7(2), 255. https://doi.org/10.17267/2317-3394rpds.v7i2.1909

Grether, E. O., Becker, M. C., Menezes, H. M., \& Nunes, C. R. d. O. (2019). Prevalência de Transtornos Mentais Comuns entre Estudantes de Medicina da Universidade Regional de Blumenau (SC). Revista Brasileira de Educação Médica, 43(1 suppl 1), 276-285. https://doi.org/10.1590/19815271v43suplemento1-20180260

Guimarães, M. F. (2014). Depressão, Ansiedade, Estresse E Qualidade De Vida De Estudantes De Universidades Pública E Privada [PublishedVersion, Universidade Metodista de São Paulo]. http://tede.metodista.br/jspui/handle/tede/1348

Ito, L. M., Ramos, R. T. Escalas de avaliação de ansiedade Rev Psiq Clin, 1998; 25(6): 294-302. Edição Especial.

Leal, R. A., dos Santos, A. L. M., da Silva, C. A., Borges, R. G. L., \& Barbosa, A. N. O. (2010). Depression and anxiety traits among medical students. Rev Paraense Med, 24(2), 13.

Leão, A. M., Gomes, I. P., Ferreira, M. J. M., \& Cavalcanti, L. P. d. G. (2018). Prevalência e Fatores Associados à Depressão e Ansiedade entre Estudantes Universitários da Área da Saúde de um Grande Centro Urbano do Nordeste do Brasil. Revista Brasileira de Educação Médica, 42(4), 55-65. https://doi.org/10.1590/1981-52712015v42n4rb20180092 
Research, Society and Development, v. 11, n. 1, e9311124556, 2022

(CC BY 4.0) | ISSN 2525-3409 | DOI: http://dx.doi.org/10.33448/rsd-v11i1.24556

Lima-Costa, Maria Fernanda, \& Barreto, Sandhi Maria. (2003). Tipos de estudos epidemiológicos: conceitos básicos e aplicações na área do envelhecimento. Epidemiologia e Serviços de Saúde, 12(4), 189-201. https://dx.doi.org/10.5123/S1679-49742003000400003

Marôco, J. P., Campos, J. A. D. B., Vinagre, M. d. G., \& Pais-Ribeiro, J. L. (2014). Adaptação Transcultural Brasil-Portugal da Escala de Satisfação com o Suporte Social para Estudantes do Ensino Superior. Psicologia: Reflexão e Crítica, 27(2), 247-256. https://doi.org/10.1590/1678-7153.201427205

Medeiros, P. P., \& Bittencourt, F. O. (2016). Fatores Associados à Ansiedade em Estudantes de uma Faculdade Particular. Id on Line Revista de Psicologia, 10(33), 42-55. https://doi.org/10.14295/idonline.v10i33.594

Melo, G. R. N., Nogueira, J. C. d. C., Macêdo, A. E. G., \& Macêdo, C. G. (2020). Transtorno de ansiedade no interior da Amazônia: um estudo de base populacional. Brazilian Journal of Development, 6(1), 5301-5311. https://doi.org/10.34117/bjdv6n1-384

Oliveira, C. T., \& Dias, A. C. G. (2014). Dificuldades na trajetória universitária e rede de apoio de calouros e formandos. Psico, 45(2), 187-197.

Oliveira, N. R. \& Barroso, S. M. (2020). Solidão, Depressão e Suporte Social em estudantes de Psicologia. Revista Trabalho EnCena, Tocantins, 5(1): 146162.

Sougey EB (1987). As escalas de avaliação nos ensaios clínicos com benzodiazepinicos - A propósito da escala de ansiedade de Hamilton. J Bras Psiquiatr $36(1), 49-53$

Souza, D. C. (2017). Condiç̃es emocionais de estudantes universitários: Estresse, depressão, ansiedade, solidão e suporte social (Dissertação de Mestrado em Psicologia). Programa de Pós Graduação em Psicologia, Universidade Federal do Triângulo Mineiro, MG. http://bdtd.uftm.edu.br/handle/tede/507

Tabalipa, F. d. O., Souza, M. F. d., Pfützenreuter, G., Lima, V. C., Traebert, E., \& Traebert, J. (2015). Prevalence of Anxiety and Depression among Medical Students. Revista Brasileira de Educação Médica, 39(3), 388-394. https://doi.org/10.1590/1981-52712015v39n3e02662014

Vilchez-Cornejo, J., Quiñones-Laveriano, D., Failoc-Rojas, V., Acevedo-Villar, T., Larico-Calla, G., Mucching-Toscano, S., Torres-Román, J. S., AquinoNúñez, P. T., Córdova-De la Cruz, J., Huerta-Rosario, A., Espinoza-Amaya, J. J., Palacios-Vargas, L. A., \& Díaz-Vélez, C. (2016). Salud mental y calidad de sueño en estudiantes de ocho facultades de medicina humana del Perú. Revista chilena de neuro-psiquiatría, 54(4), 272-281. https://doi.org/10.4067/s071792272016000400002

Vizzotto, M. M., Jesus, S. N. d., \& Martins, A. C. (2017). Saudades de Casa: indicativos de depressão, ansiedade, qualidade de vida e adaptação de estudantes universitários. Revista Psicologia e Saúde. https://doi.org/10.20435/pssa.v9i1.469 